\title{
Nondestructive Quantification of Analyte Diffusion in Cornea and Sclera Using Optical Coherence Tomography
}

\author{
Mohamad G. Ghosn, ${ }^{1}$ Valery V. Tuchin, ${ }^{2}$ and Kirill V. Larin ${ }^{1,2}$
}

Purpose. Noninvasive functional imaging, monitoring, and quantification of analytes transport in epithelial ocular tissues are extremely important for therapy and diagnostics of many eye diseases. In this study the authors investigated the capability of optical coherence tomography (OCT) for noninvasive monitoring and quantification of diffusion of different analytes in sclera and cornea of rabbit eyes.

Methods. A portable time-domain OCT system with wavelength of $1310 \pm 15 \mathrm{~nm}$, output power of $3.5 \mathrm{~mW}$, and resolution of $25 \mu \mathrm{m}$ was used in this study. Diffusion of different analytes was monitored and quantified in rabbit cornea and sclera of whole eyeballs. Diffusion of water, metronidazole $(0.5 \%)$, dexamethasone $(0.2 \%)$, ciprofloxacin $(0.3 \%)$, mannitol (20\%), and glucose solution (20\%) were examined, and their permeability coefficients were calculated by using OCT signal slope and depth-resolved amplitude methods.

Results. Permeability coefficients were calculated as a function of time and tissue depth. For instance, mannitol was found to have a permeability coefficient of $(8.99 \pm 1.43) \times 10^{-6} \mathrm{~cm} / \mathrm{s}$ in cornea and $(6.18 \pm 1.08) \times 10^{-6} \mathrm{~cm} / \mathrm{s}$ in sclera. The permeability coefficient of drugs with small concentrations (where water was the major solvent) was found to be in the range of that of water in the same tissue type, whereas permeability coefficients of higher concentrated solutions varied significantly.

Conclusions. Results suggest that the OCT technique might be a powerful tool for noninvasive diffusion studies of different analytes in ocular tissues. However, additional methods of OCT signal acquisition and processing are required to study the diffusion of agents of small concentrations. (Invest Ophthalmol Vis Sci. 2007;48:2726-2733) DOI:10.1167/iovs.06-1331

$\mathrm{D}$ iseases affecting posterior segment of the eye, such as age-related macular degeneration (AMD), diabetic retinopathy, glaucoma, and retinitis pigmentosa, are the principal causes of irreversible blindness worldwide. ${ }^{1,2}$ AMD alone affects approximately $\mathbf{1 . 7}$ million people in the United States, and this number will grow as the nation ages. ${ }^{3}$ Successful management of these diseases requires long-term treatment

From the ${ }^{1}$ Biomedical Engineering Program, University of Houston, Houston, Texas; and the ${ }^{2}$ Institute of Optics and Biophotonics, Saratov State University, Saratov, Russia.

Supported by Federal Agency of Education of RF Grants 1.4.06 and RNP.2.1.1.4473 (VVT) and by CRDF BRHE Grant RUXO-006-SR-06 (VVT).

Submitted for publication November 6, 2006; revised December 15, 2006; accepted March 26, 2007. None

Disclosure: M.G. Ghosn, None; V.V. Tuchin, None; K.V. Larin,

The publication costs of this article were defrayed in part by page charge payment. This article must therefore be marked "advertisement" in accordance with 18 U.S.C. $\$ 1734$ solely to indicate this fact.

Corresponding author: Kirill V. Larin, Biomedical Engineering Program, University of Houston, N207 Engineering Building 1, Houston, TX 77204; klarin@uh.edu. with drugs. Topical and transscleral drug delivery are the least invasive and the most common ways to treat anterior eye diseases. ${ }^{4}$ However, the delivery of therapeutic agents to target tissues in effective concentrations remains difficult (and potentially dangerous) because of the low permeability of ocular tissues and drug washout. ${ }^{3}$ Significant recent advances have optimized the delivery of drugs to target tissues within the eye for the pharmacologic treatment and diagnostics of many ocular diseases. ${ }^{5}$ However, diffusion constants of therapeutic agents in these tissues in vivo remain unknown, primarily because of the lack of a noninvasive imaging modality capable of real-time monitoring and quantification of drug diffusion.

Additionally, functional imaging, monitoring, and quantification of analyte diffusion across epithelial and stromal ocular tissues are extremely important for therapy and diagnostics of many eye diseases. Efficient therapy requires proper drug selection and effective drug delivery. Designing effective formulations of drugs requires knowledge of its diffusion permeability across epithelial tissues in vivo. Moreover, changes in diffusion permeability of different chemical compounds and organic solutions may help in the diagnosis of different ocular diseases and abnormalities.

In the past decades, several scientific groups have been developing and applying different experimental techniques to study drug diffusion in tissues in vitro, including spectrofluorometers and Ussing apparatus, ${ }^{6-8}$ fluorescence microscopy, ${ }^{9,10}$ and microdialysis (Mitra AK, et al. IOVS 2001;42: ARVO Abstract 174). Permeability of sclera, cornea, and other ocular tissues for different molecular weight compounds was summarized by Prausnitz et al. ${ }^{11}$ These techniques have enriched our knowledge of drug diffusion properties in ocular tissues; however, they are limited to in vitro studies only.

Recently, magnetic resonance imaging (MRI) has been proposed for in vivo imaging of drug diffusion in eyeballs. ${ }^{12,13}$ Results suggest that MRI might be a powerful tool for imaging and quantification of drug distribution inside the eye globe. However, several drawbacks of this technology (such as the limited availability of actual drugs with paramagnetic properties, length of time needed for image acquisition and signal processing, and low resolution) limit its application for drug diffusion studies in epithelial tissues, such as cornea and sclera.

Optical coherence tomography (OCT) is a relatively new noninvasive, optical, diagnostic technique that provides depthresolved images of tissues with resolution up to a few micrometers and depths up to several millimeters (depending on tissue type). Since its introduction in $1991,{ }^{14}$ this technique has been extensively applied in many areas of biomedicine. ${ }^{15-17}$ The basic principle of the OCT is to detect backscattered photons from a tissue of interest within a coherence length of the source using a two-beam interferometer. The use of broadband laser sources and varying optical path lengths in the sample arm allows light intensity to be recorded as a function of depth. By gathering interference data at points across the surface, two-dimensional and three-dimensional images can be formed. 
Low-coherence interferometry (LCI), a modality of OCT, has been previously used for monitoring of dye diffusion in agar gel. ${ }^{18}$ Changes in the optical properties of the gel sample during the diffusion process of a dye were monitored using dual-wavelength LCI. The method is based on differential measurements of the gel's optical properties at the two probing wavelengths, where one is unaffected and the other is strongly absorbed by the dye. Recently, we also demonstrated the capability of single-source OCT for monitoring of glucose diffusion in tissues based on time- and depth-resolved analysis of the scattering properties of tissues. ${ }^{19}$ These results suggest that the OCT technique could be successfully applied for functional imaging and quantification of diffusion processes in tissuesimulating objects and real tissues.

In this article, we report the results of our pilot studies on the application of OCT for monitoring and quantifying the diffusion of different analytes in the sclera and cornea of whole rabbit eyes ex vivo.

\section{Materials ANd Methods}

\section{Principles of Drug-Induced Changes in Optical Properties of Tissue}

An important ability of a chemical compound (agent) to change tissuescattering properties is based on a number of biophysical processes. $^{20-26}$ The concept of two fluxes-agent into tissue and bulk tissue water out-that may be relatively independent or interacting fluxes defines the dynamic properties of tissue optical clearing and its efficiency. ${ }^{26}$ Optical clearing agent (OCA) diffusion inside tissue to the interstitial space leads to an increase of the refractive index of the interstitial fluid (ISF) and thus to refractive index matching of collagen fiber (and other tissue components, or scatterers) and ISF. A hyperosmotic OCA may induce intensive water flow from tissue and may cause its strong dehydration and corresponding alteration of tissue morphologic and optical properties, as follows: refractive index matching/ mismatching (generally for whole tissue matching; local mismatching may take place), increased tissue collagen fiber packing density (ordering of scatterers), and decreased tissue thickness. Tissue collagen reorganization also may take place. $^{24}$
The scattering coefficient of tissues depends on the refractive index, $n$, mismatch between the ISF ( $\left.n_{\text {ISF }}\right)$ and the tissue components (fibers, cell components) $\left(n_{\mathrm{s}}\right)$. If the refractive index of the scattering centers $n_{\mathrm{s}}$ remains constant and is higher than the refractive index of the medium, the addition of the agent with refractive index $\delta n_{\text {agent }}$ to surrounding medium reduces the refractive index mismatch, $n_{\mathrm{s}}-\left(n_{\mathrm{ISF}}\right.$ $\left.+\delta n_{\text {agent }}\right)$. In a simple model of scattering dielectric spheres, the reduced scattering coefficient, $\mu_{\mathrm{s}}^{\prime}=\mu_{\mathrm{s}}(1-g)$, where $g$ is the tissue anisotropy factor, can be approximated $\mathrm{as}^{27}$ :

$$
\mu_{\mathrm{s}}^{\prime}=3.28 \pi r^{2} \rho_{\mathrm{s}}\left(\frac{2 \pi r}{\lambda}\right)^{0.37}\left(\frac{n_{\mathrm{s}}}{n_{\mathrm{ISF}}+\delta n_{\text {agent }}}-1\right)^{2.09}
$$

where $r$ is the sphere radius, $\rho_{\mathrm{s}}$ the volume density of the spheres, and $\lambda$ the wavelength of the incident light. Therefore, increased tissue drug concentration will raise the refractive index of the surrounding me$\operatorname{dium}\left(n_{\text {ISF }}+\delta n_{\text {agent }}\right)$, which will decrease the scattering coefficient of the tissue as a whole.

Alternatively, the change in local concentrations of scattering particles, such as cell components or collagen fibers, could change scattering as well. Tissue mean refractive index can be calculated by the law of Gladstone and Dale as a weighted average of refractive indices of modified interstitial fluid ( $\left.n_{\mathrm{ISF}}^{\prime}=n_{\mathrm{ISF}}+\delta n_{\text {agent }}\right)$ and collagen fibers or cell components $\left(n_{\mathrm{c}}\right)$ :

$$
\bar{n}=\phi_{\mathrm{c}} n_{\mathrm{c}}+\left(1-\phi_{\mathrm{c}}\right) n_{\mathrm{ISF}}^{\prime},
$$

where $\phi_{\mathrm{c}}$ is the volume fraction of collagen fibers or cell components in tissues. Therefore, changes in the volume fraction of tissue components $\phi_{\mathrm{c}}$ (e.g., by shrinkage or swelling of the tissues) will change the overall refractive index of the tissues.

Because the OCT technique measures in-depth intensity distribution with high resolution, changes in the in-depth distribution of the tissue-scattering coefficient or refractive index are reflected in changes in the OCT signal. ${ }^{21-23,28-31}$ Thus, because the diffusion of agents in tissues introduces local changes in their optical properties (scattering coefficient and local and mean refractive indices), one can monitor and quantify the diffusion process by analyzing the changes in the OCT signal slope and the amplitude recorded from a sample.

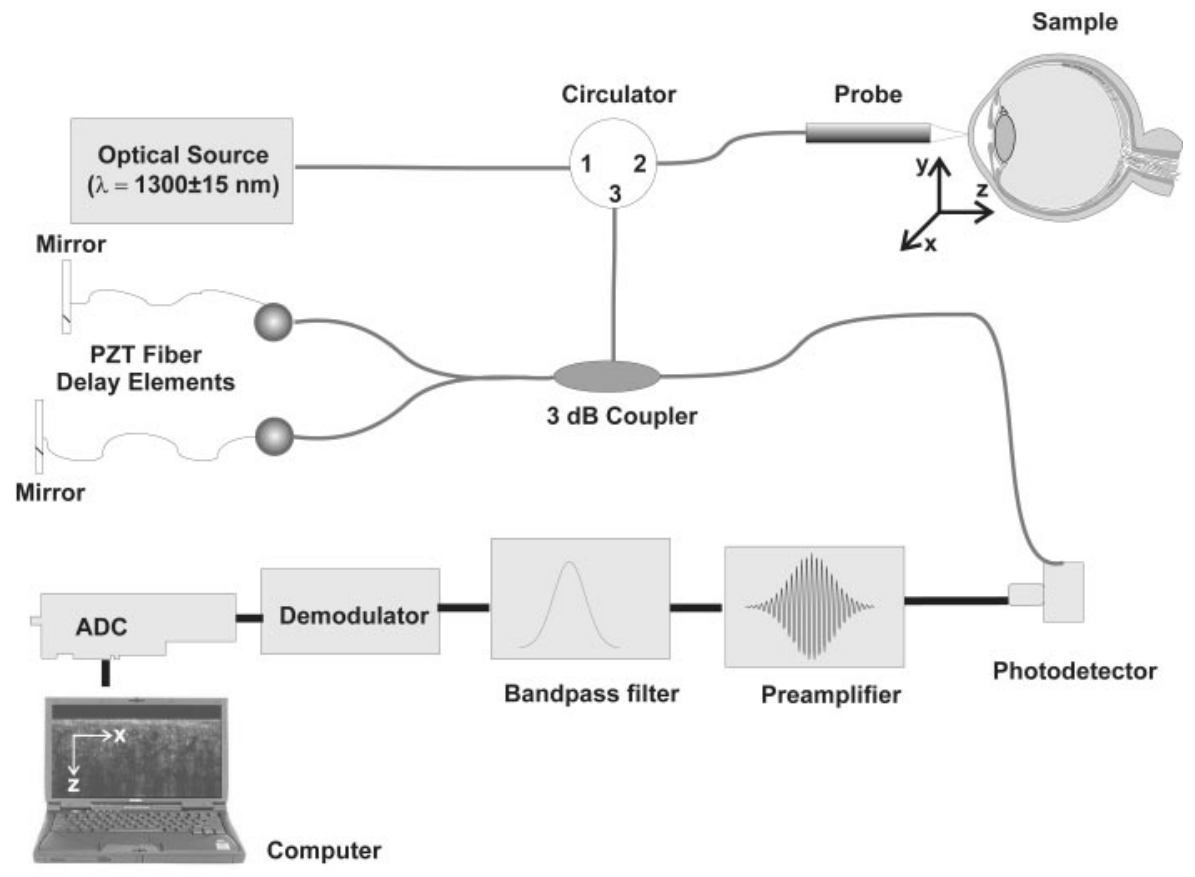

Figure 1. Schematic diagram of experimental setup. 


\section{Experimental Setup}

Experiments were performed with a portable time-domain OCT system (Imalux Corp., Cleveland, OH; Fig. 1). The optical source used in this system was a low-coherent broadband, near-infrared (NIR) light source with wavelength of $1310 \pm 15 \mathrm{~nm}$, output power of $3.5 \mathrm{~mW}$, and resolution of $25 \mu \mathrm{m}$ (in air). Light in the sample arm of the interferometer was directed to the tissues with a single-mode optical fiber and a specially designed miniature endoscopic probe. The endoscopic probe allowed lateral scanning of the sample surface in the lateral direction ( $x$-axis). Light scattered from the sample and light reflected from the reference arm mirror formed an interferogram, which was detected by a photodiode. In-depth scanning was produced electronically by piezoelectric modulation of the fiber length. Two-dimensional images were obtained by scanning the incident beam over the sample surface in the lateral direction and in-depth (z-axial) scanning by the interferometer (Figs. 2a, 2c). Acquired images measured $2.2 \times 2.4 \mathrm{~mm}$. The full image acquisition rate was approximately 3 seconds per image. The operation of the OCT system was fully controlled by PC. The two-dimensional images were averaged in the lateral direction ( $x$-axis) (over approximately $1 \mathrm{~mm}$, which was sufficient for speckle-noise suppression) into a single curve to obtain an OCT signal that represented one-dimensional distribution of light in-depth in logarithmic scale (Figs. 2b, 2d). The depth scales shown in Figures $2 \mathrm{~b}$ and $2 \mathrm{~d}$ and in similar graphs thereafter were calculated by dividing the optical path length given by the OCT system to the mean refractive index of the tissue, which was assumed 1.4 in the sclera and the cornea.

\section{Tissue Samples}

Experiments were performed with fresh New Zealand white rabbit eyes (obtained from Vision Tech Inc., Dallas, TX). Eyes were transported to our laboratory while kept cool and were stored in $0.9 \%$ sodium chloride solution to prevent dehydration. They were freshly enucleated, and experiments were performed within the first three days of arrival to guarantee minimal changes in the physiological status of the tissues. Before experiments, the eyes were taken out of their a)
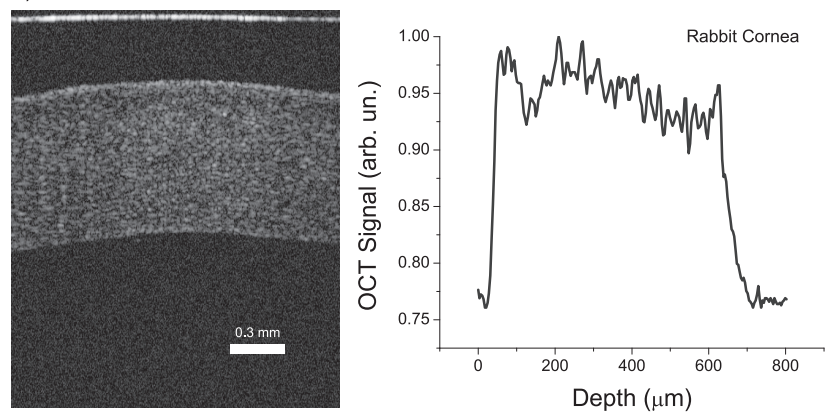

c)

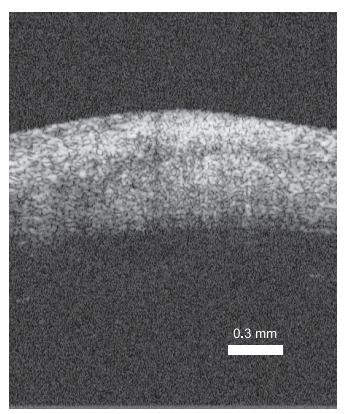

d)

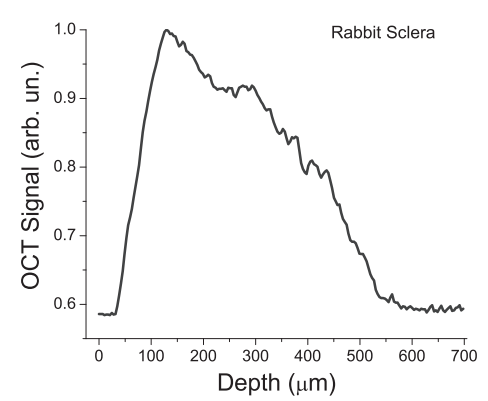

FIGURE 2. Typical OCT image and corresponding one-dimensional signal recorded from rabbit cornea $(\mathbf{a}, \mathbf{b})$ and rabbit sclera $(\mathbf{c}, \mathbf{d})$. cooled storage environment and placed for an hour in a specially designed dish containing saline solution. One hour was sufficient for the eye to reach room temperature. Experiments were conducted at $22^{\circ} \mathrm{C}$. Constant temperature was maintained for the duration of the experiments. OCT images were continuously acquired for 60 to 180 minutes. The laser beam was delivered perpendicularly to the epithelial surface of the tissue (sclera or cornea). Before any agent was added, images of the sclera or the cornea were taken for approximately 8 to 10 minutes to record a baseline.

\section{Agents}

The diffusion of different drugs and solutions was studied during these experiments. Purified and distilled water was monitored during the diffusion process, and the permeability coefficient was calculated. Diffusion of $20 \%$ glucose solution was also studied. Glucose solutions were prepared by dissolving different amounts $(20 \mathrm{~g}$ and $40 \mathrm{~g})$ of glucose (Mallinckrodt Baker, Inc., Phillipsburg, NJ) in $100 \mathrm{~mL}$ pure water to obtain $20 \%$ and $40 \%$ glucose solutions, respectively.

We studied the diffusion of several ophthalmic drugs, including metronidazole, ciprofloxacin, dexamethasone, and mannitol. These drugs are widely used in ophthalmology and are administered topically. The concentrations of metronidazole (Abbott Laboratories, North Chicago, IL), ciprofloxacin (Alcon Laboratories, Fort Worth, TX), dexamethasone (Phoenix Scientific, Inc., St. Joseph, MO), and mannitol were $0.5 \%, 0.3 \%, 0.2 \%$, and $20 \%$, respectively.

\section{Data Processing}

The permeability coefficient of the drugs and solutions in cornea and sclera was calculated using two methods, OCT signal slope (OCTSS) and OCT amplitude (OCTA) measurements with a specially developed $\mathrm{C}++$ program. With the OCTSS method, the average permeability coefficient of a specific region in the tissue was calculated by analyzing the slope changes in the OCT signal caused by analyte diffusion. Two-dimensional OCT images were averaged in the lateral ( $x$-axial) direction into a single curve to obtain an OCT signal that represented the one-dimensional distribution of intensity in-depth (in logarithmic scale; Fig. 3). A region in the tissue, where the signal was linear and underwent minimal alterations, was selected, and its thickness $\left(z_{\text {region }}\right)$ was measured. Diffusion of the agents in the chosen region was monitored, and time of diffusion was recorded $\left(t_{\text {region }}\right)$. The average permeability coefficient $(\overline{\mathrm{P}})$ was calculated by dividing the measured thickness of the selected region by the time it took for the agent to diffuse through $\left(\overline{\mathrm{P}}=\frac{z_{\text {region }}}{t_{\text {region }}}\right)$.

The OCTA method was used to calculate the permeability coefficient at specific depths in the tissues as $\mathrm{P}(z)=z_{\mathrm{i}} / t_{z_{i}}$, where $z_{\mathrm{i}}$ was the depth at which measurements were performed (calculated from the front surface) and $t_{z_{i}}$ was the time of agent diffusion to this depth. The $t_{z_{i}}$ was calculated from the time agent was added to the tissue until agent-induced change in the OCT amplitude was commenced. Note that unlike in OCTSS method, the $z_{\mathrm{i}}$ was calculated from the epithelial surface of the tissues to the specific depth in tissue stroma in an OCTA method.

\section{Results}

\section{Diffusion in Cornea}

Figure 4 shows a typical OCTSS graph for a rabbit cornea during a dexamethasone diffusion experiment. The bottom hemisphere of the eyeball was submersed in saline solution to prevent dehydration of the eye. The OCTSS was calculated to be 88 to $368 \mu \mathrm{m}$ regions from the surface of the cornea. First, the cornea was monitored for approximately 8 minutes before the drug was added. A single droplet of dexamethasone was applied, and monitoring of the cornea continued for another 60 minutes. The propagation of dexamethasone inside the 


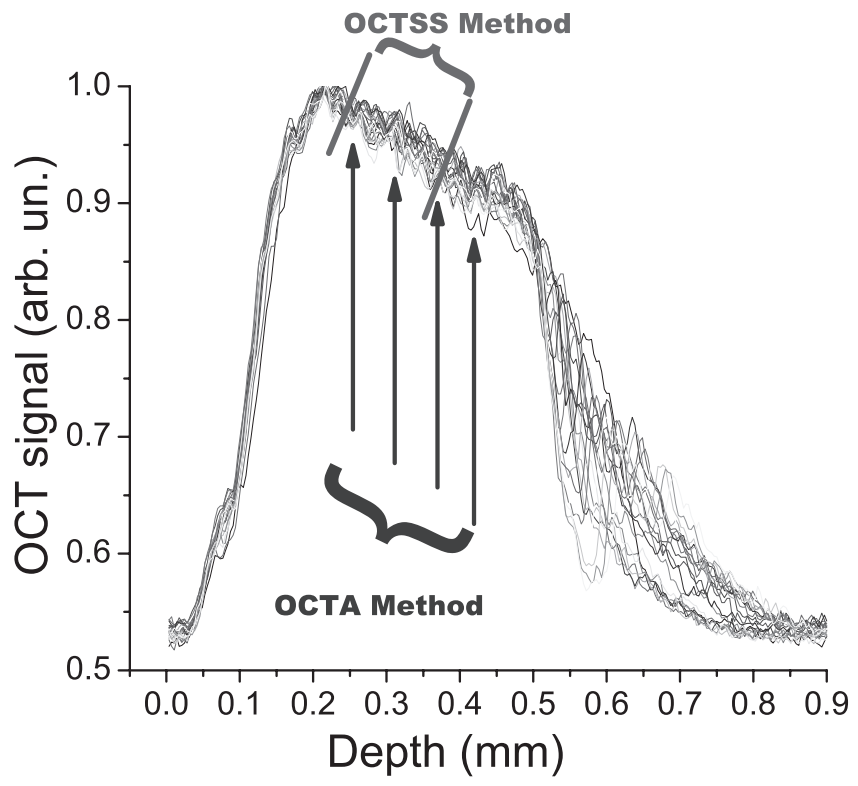

FigURE 3. OCT signals recorded from a rabbit sclera at different times indicating region (approximately $130 \mu \mathrm{m}$ ) for OCTSS measurement and representative depths (at approximately 105, 160, 220, and 270 $\mu \mathrm{m}$ from the epithelial layer) for OCTA measurements.

cornea changed the local scattering coefficient and was detected by the OCT system. The increase in local in-depth concentration of dexamethasone resulted in the decrease of scattering coefficient and hence in the decrease of the OCT signal slope. After approximately 15 minutes from application, the droplet of dexamethasone had entirely penetrated through the tissue. When the drug reached the aqueous humor, a reverse process took place. From the physiology of the cells and tissues, concentration gradient between two sides of a tissue created a driving force for a fluid to travel from the medium of high concentration to that of lower one. This force is likely to be the source of the reverse process seen in Figure 4. The driving force stays active until equilibrium is reached.

Permeability coefficients of different agents in cornea were measured in 28 experiments. The permeability coefficient for water was found to be $(1.68 \pm 0.54) \times 10^{-5} \mathrm{~cm} / \mathrm{s}$. Ciprofloxacin $(0.3 \%)$, dexamethasone $(0.2 \%)$, and metronidazole $(0.5 \%)$ had permeability coefficients of $(1.85 \pm 0.27) \times 10^{-5},(2.42 \pm$ $1.03) \times 10^{-5}$, and $(1.59 \pm 0.43) \times 10^{-5} \mathrm{~cm} / \mathrm{s}$, respectively. A summary of the permeability coefficients of different agents in the rabbit cornea is presented in Table 1.

The diffusion constant of water in rabbit cornea of $1.5 \times$ $10^{-4} \mathrm{~cm} / \mathrm{s}$ was reported by Grass ${ }^{32}$ based on in vitro measurements on isolated tissues. Ciprofloxacin was stated to have a permeability coefficient of $0.29 \times 10^{-5} \mathrm{~cm} / \mathrm{s}^{33}$ This discrepancy between previously reported and measured by using OCT values for water and ciprofloxacin diffusion in cornea most likely results from distinctions in experimental and calculation methods used for estimation of the permeability coefficients and for tissue preparation (e.g., isolated versus intact tissues, hydration factors). Recently, we demonstrated that the permeability coefficients of several agents might be different if they were measured in isolated cornea or cornea in whole eyeball (e.g., calculated permeability coefficient of pure water in isolated cornea was $1.39 \pm 0.24 \times 10^{-4} \mathrm{~cm} / \mathrm{s}$, which is very close to the previously reported values). ${ }^{34}$

\section{Diffusion in Sclera}

Figure 5 demonstrates a typical OCTSS graph obtained from a rabbit sclera during a glucose diffusion experiment. This experiment was conducted while the whole eyeball was fully submersed in the $20 \%$ glucose solution. First, the eyeball was imaged for 10 minutes while placed in $4 \mathrm{~mL}$ water. Another 4 $\mathrm{mL}$ of $40 \%$ glucose was added to make $8 \mathrm{~mL}$ of $20 \%$ concentrated glucose solution. The OCT signal slope was calculated from a $105-\mu \mathrm{m}$ thick region $(70-175-\mu \mathrm{m}$ depth from the surface of the sclera). The increase in the local in-depth concentration of glucose in sclera initiated a decrease in the scattering coefficient and the OCTSS. The same reverse process occurred in these experiments. The amplitude and speed of

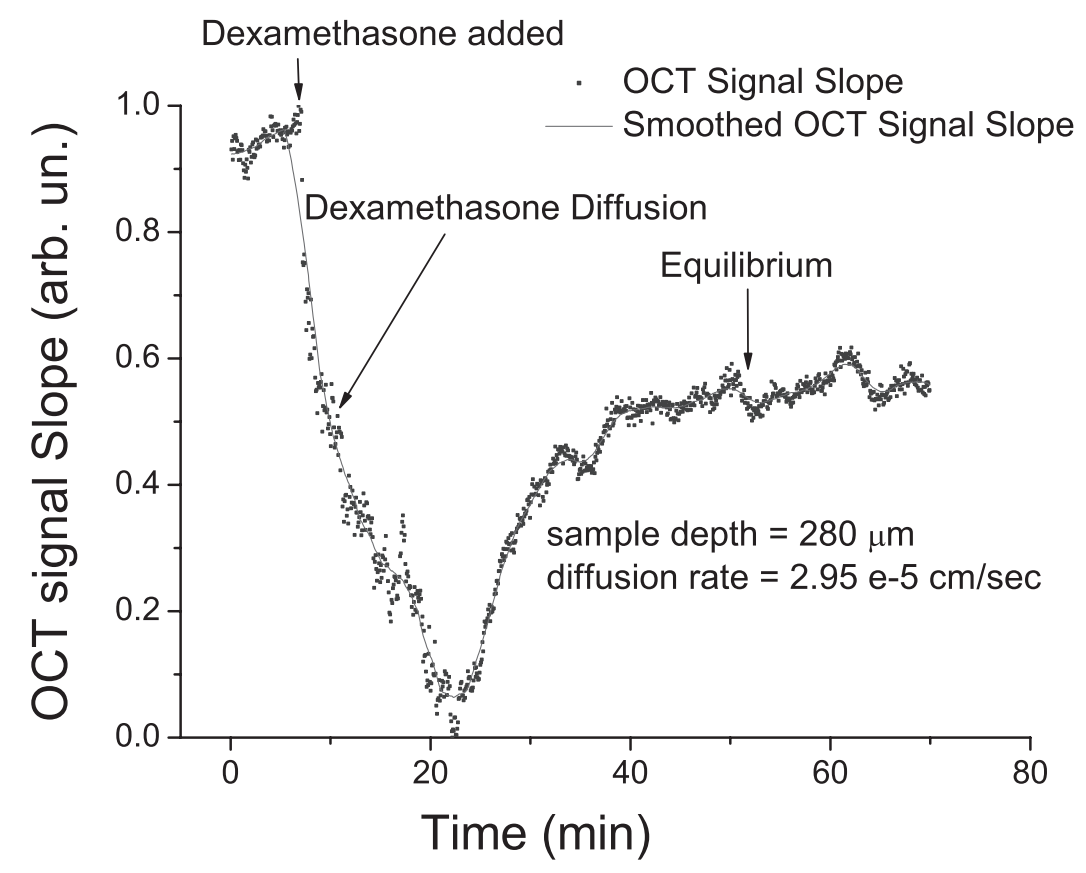

Figure 4. OCT signal slope as a function of time recorded from rabbit cornea during dexamethasone diffusion in partially submersed eyeball experiments. 
TABLE 1. Permeability Coefficient of Agents in Rabbit Cornea

\begin{tabular}{lcc}
\hline \multicolumn{1}{c}{ Agent } & $\begin{array}{c}\text { Permeability Coefficient } \pm \text { SD } \\
(\mathbf{c m} / \mathbf{s})\end{array}$ & $\begin{array}{c}\text { No. of Independent } \\
\text { Experiments }\end{array}$ \\
\hline Water & $(1.68 \pm 0.54) \times 10^{-5}$ & 8 \\
Ciprofloxacin & $(1.85 \pm 0.27) \times 10^{-5}$ & 4 \\
Mannitol & $(8.99 \pm 1.43) \times 10^{-6}$ & 4 \\
Dexamethasone & $(2.42 \pm 1.03) \times 10^{-5}$ & 7 \\
Metronidazole & $(1.59 \pm 0.43) \times 10^{-5}$ & 5 \\
\hline
\end{tabular}

the reversal process could be governed by the volume-tovolume ratio between the eye and the solution in which it is residing in. The volume of the eye was estimated to be $8 \mathrm{~cm}^{3}$. For that, we maintained $8-\mathrm{cm}^{3}$ volume of drug solutions to keep a 1:1 ratio. As the result, the equilibrium stage was reached at approximately $50 \%$ of initial value.

Thirty-one experiments were performed on sclera of different rabbit eyes. The permeability coefficient of water was found to be $(1.33 \pm 0.28) \times 10^{-5} \mathrm{~cm} / \mathrm{s}$. Ciprofloxacin, mannitol, glucose $20 \%$, and metronidazole had permeability coefficients of $(1.41 \pm 0.38) \times 10^{-5},(6.18 \pm 1.08) \times 10^{-6},(8.64 \pm$ $1.12) \times 10^{-6}$, and $(1.31 \pm 0.31) \times 10^{-5} \mathrm{~cm} / \mathrm{s}$, respectively. Results of the permeability coefficients of these agents in sclera are summarized in Table 2 . These results match coefficients published in earlier studies. The permeability coefficient of ciprofloxacin in rabbit sclera was measured as (1.88 \pm $0.617) \times 10^{-5} \mathrm{~cm} / \mathrm{s}^{33}$ Previously, mannitol (approximately $0.3 \%)$ was reported to have a permeability coefficient of $(2.6 \pm$ $0.9) \times 10^{-5} \mathrm{~cm} / \mathrm{s}$ in rabbit sclera from a magnetic resonance imaging study. ${ }^{35}$ The difference between these values are likely attributed to the difference in the concentrations and in experimental conditions. However, results for glucose diffusion are in a good correlation with those for human sclera. ${ }^{36}$

\section{In-depth Diffusion Monitoring}

The OCT technique has some unique properties that distinguish it from other imaging techniques. Among those are high resolution and ability for noninvasive depth-resolved imaging. These properties allowed investigation of the diffusion of ana- lytes not only as a function of time but also as a function of depth. Using the OCT system, we monitored the diffusion process of different analytes at various depths away from the surface of the tissue. Figure 6 shows a typical OCT signal measured at depths $105,158,225$, and $273 \mu \mathrm{m}$ away from the surface of a rabbit sclera during a mannitol diffusion experiment. The arrows on each of the OCT signals depict the time the drug action reached that particular depth (manifested as sharp decrease of the OCT signal). Figure 7 shows the permeability coefficients of $20 \%$ mannitol recorded at different depths of the sclera and calculated using the OCTA method. Mannitol was added at 6 th minute in this experiment. This graph demonstrated that permeability coefficient inside the sclera is not uniform and is increasing with increase of the depth.

\section{Discussion}

Results shown in Figures 4 to 7 and summarized in Tables 1 and 2 demonstrate the ability of the OCT technique for accurate monitoring and quantification of agents' diffusion in epithelial eye tissues. Diffusion of different agents in cornea and sclera locally changed tissues' optical properties that were depicted by depth-resolved analysis of the OCT signals. Additionally, one can see from these tables that the permeability coefficients of small-concentrated agents dissolved in water (ciprofloxacin- $0.3 \%$ and metronidazole-0.5\%) lie within the range of that of pure water. Hence, the changes in the optical properties of the tissues were induced, most likely by the

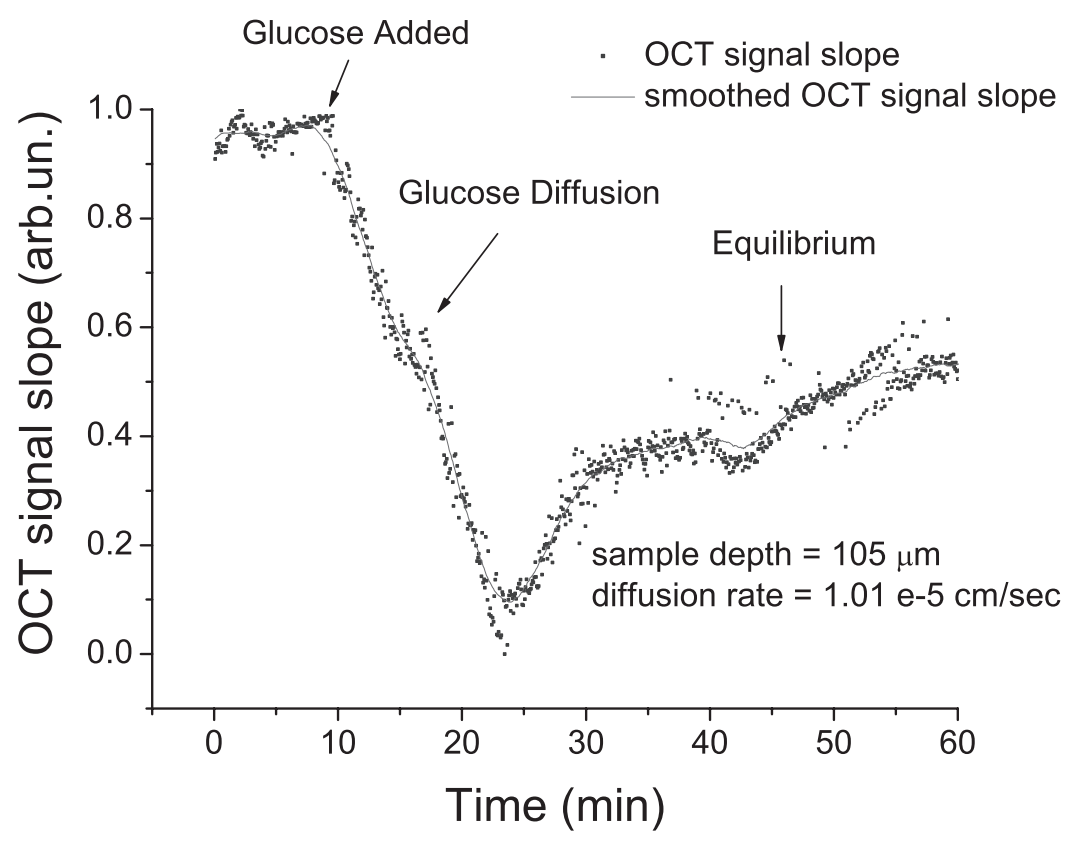

FIGURE 5. OCT signal slope as a function of time recorded from sclera during glucose $20 \%$ diffusion in fully submersed eyeball experiments. 
TABle 2. Permeability Coefficient of Agents in Rabbit Sclera

\begin{tabular}{lcc}
\hline \multicolumn{1}{c}{ Agent } & $\begin{array}{c}\text { Permeability Coefficient } \pm \text { SD } \\
(\mathbf{c m} / \mathbf{s})\end{array}$ & $\begin{array}{c}\text { No. of Independent } \\
\text { Experiments }\end{array}$ \\
\hline Water & $(1.33 \pm 0.28) \times 10^{-5}$ & 5 \\
Ciprofloxacin & $(1.41 \pm 0.38) \times 10^{-5}$ & 3 \\
Mannitol & $(6.18 \pm 1.08) \times 10^{-6}$ & 5 \\
Glucose & $(8.64 \pm 1.12) \times 10^{-6}$ & 14 \\
Metronidazole & $(1.31 \pm 0.29) \times 10^{-5}$ & 4 \\
\hline
\end{tabular}

carrier of the drug solution rather than the drug itself in this case. Permeability coefficients for the small-concentrated dexamethasone $(0.2 \%)$ were significantly different from that of water because of a distinct carrier-polyethylene glycol 400 of $50 \%$ concentration. Nevertheless, monitoring and quantification of diffusion processes of small-concentrated agents in tissues might be possible by application of different methods of OCT signal acquisition and processing. For example, as mentioned, the diffusion of drugs in tissues might affect its collagen organization..$^{8,24,37-40}$ One of the most effective noninvasive methods for monitoring the structural rearrangements is polarization-sensitive (PS) OCT. ${ }^{41-44}$ OCT images in orthogonal polarization can potentially provide higher sensitivity and specificity of drug diffusion monitoring. Another approach might be the application of drugs in different formulations (e.g., ointments, gels, and various sustained and controlled-release substrates).

Comparison of results summarized in Tables 1 and 2 demonstrates that permeability coefficients for the same analyte in the cornea and sclera are different. This discrepancy is likely the result of structural and physiological differences. Hydration is also considered a key factor in the study of permeability coefficients of agents through biological tissues. With hydration, the fibrils move further apart, creating extra space between them. ${ }^{45}$ Swelling could come from the glycosaminoglycans (GAGs) or from anion bindings occurring in the tissue. ${ }^{46,47}$ The sclera has 10 times fewer GAGs than the cornea, and the scleral stroma has a greater degree of fibrillar interweave than corneal stroma. ${ }^{48}$ Thus, even though the cornea swells to many times its original weight in aqueous solution, the hydration of sclera increases only $20 \%$ to $25 \% .{ }^{46}$ It has been demonstrated that the permeability coefficient is propor-

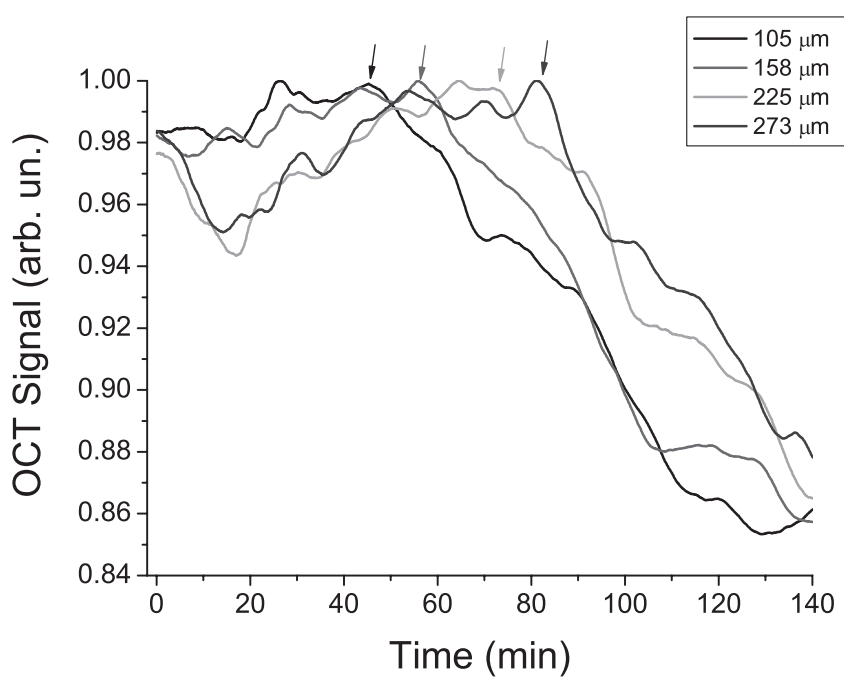

FIGURE 6. OCT signal as a function of time recorded at different depths during a mannitol diffusion experiment in the sclera. Arrows indicate the mannitol front reaching a different depth inside sclera. tional to the tissues' hydration state. As the hydration in a tissue increases, the permeability coefficient increases. ${ }^{49}$ In our experiments, whole rabbit eyeballs were kept hydrated by placing each bottom hemisphere or complete eyeball in a saline solution (to minimize the natural dehydration processes caused by surface evaporation). Thus, the cornea and sclera were slightly overhydrated compared with natural conditions. This could be the reason for the higher permeability coefficient in cornea than in sclera of the same agent and the difference between in vitro and limited in vivo data. Given that the most natural tissue hydration state can be realized only during in vivo studies, future animal studies will provide tangible permeability coefficients of different drugs and agents in ocular tissues. The hydration state of tissues also affects local and average refractive index of tissues. In this study, the refractive index was assumed to be 1.4 for all calculations of the permeability coefficients. This assumption is not entirely accurate because agent diffusion will change the tissue refractive index and, therefore, will affect the size of the tissue region used for the calculations. This change in tissue optical size was small enough and was neglected in our calculations. However, we will develop correction models for the change of local refractive index and estimate its influence on the accuracy of permeability coefficient calculations in our future studies, including application of Raman spectroscopy for simultaneous assessment of agent diffusion and of the local hydration state of ocular tissue.

Results of depth-resolved quantification of agent diffusion in tissues, shown in Figures 6 and 7 , demonstrated that the permeability coefficient inside epithelial tissues is increased with the increase in depth. Several factors contribute to this increase. The layered structure of the tissues, the difference in the diameter sizes of the collagen fibers in each layer, and the diverse organizational patterns of the collagen bundles at different depths are likely to contribute to the observed trend. For instance, the sclera has three distinct layers, the episclera, the

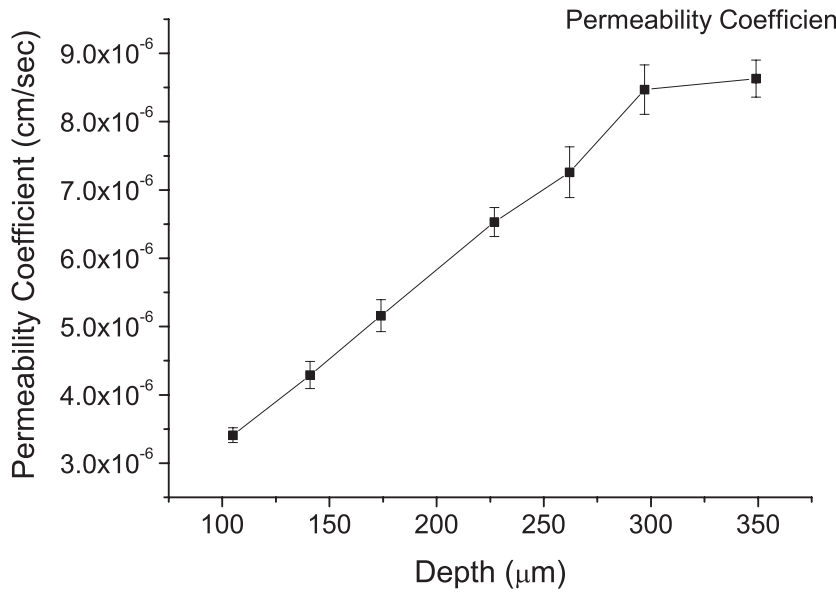

FiguRe 7. Permeability coefficients recorded at different depths in a sclera during the diffusion of $20 \%$ mannitol. 
stroma, and the lamina fusca. The outer layer, the episclera, primarily consists of collagen bundles that intersect at different angles along the surface of the sclera. That inconsistency of the organization of the collagen bundles imposes a resistance force that reduces the speed of penetration of the agents to the tissue, resulting in lower permeability coefficient of the agent. In the stroma and the lamina fusca, the collagens are more organized and oriented in two patterns, meridional and circular, contributing to increased permeability in the stroma. Additionally, the collagen bundles differ in size throughout the different layers of the sclera: the collagen bundles in the external region of the sclera are narrower and thinner than those in the inner region. ${ }^{50}$ The different diameters of the collagen fibers in different tissue depths might also influence the diffusion in tissues.

\section{Conclusions}

The experimental results obtained in these studies demonstrate that OCT can be an effective tool in the study of agent diffusion through tissues. Different analytes and chemical compounds, such as water, glucose, ciprofloxacin, dexamethasone, metronidazole, and mannitol, were tested, and corresponding permeability coefficients were calculated for the sclera and the cornea. However, calculated permeability coefficients for small-concentrated drugs are very close to those of water, suggesting the necessity of additional studies. Permeability coefficients were computed as a function of time and a function of depth. The differences in the carriers and in the physical, chemical, and solubility properties of these analytes contribute to the differences in the measured permeability coefficients.

Results obtained from our experiments showed close correlation with those published in previous studies. Even though different methods and techniques were applied in diffusion experiments, the numerical values seem to be consistent. Indepth permeability coefficients were also estimated in the sclera and the cornea. An increase in the permeability coefficient as a function of depth was observed. This increase was likely caused by the layered organization of the tissue. The capability of in-depth measurements poses a new trend in the study of drug diffusion and might be beneficial and of a great importance in different fields.

Diffusion across the sclera has been studied extensively as a function of molecular weight and other parameters. Recently, Prausnitz et al. ${ }^{51}$ examined diffusion within the sclera in the lateral direction for its possible effect in drug distribution. Administering drugs in the lateral direction could present a new trend in retinal diseases treatment. The results demonstrated in this article demonstrate the possibility of noninvasive depth-resolved quantification of agent diffusion in cornea and sclera. Application of three-dimensional OCT can easily extend these results for noninvasive quantification of agent lateral diffusion in eye tissues.

Additionally, nondestructive and potentially noninvasive studies of agent diffusion in tissues might be significant if the agents are part of pharmaceutical and diagnostic analysis. Advanced formulations of different drugs might depend on the diffusion studies. Moreover, the permeability coefficient of certain drugs and solutions in tissues might play a role in diagnosing diseases and abnormalities. Several pathologic and disease conditions can alter the mechanical properties of the extracellular matrix (ECM). For example, corneal abnormalities such as stromal matrix structural irregularity are a lead aspect of cancer and fibrosis. ${ }^{52}$ These and many other diseases might be diagnosed by comparing the permeability coefficients of normal and abnormal tissues.

\section{References}

1. Quigley HA. Number of people with glaucoma worldwide. $\mathrm{Br} J$ Ophthalmol. 1996;80:389-393.

2. Starr CE, Guyer DR, Yannuzzi LA. Age-related macular degeneration: can we stem this worldwide public health crisis? Postgrad Med. 1998;156:161-164.

3. Geroski DH, Edelhauser HF. Drug delivery for posterior segment eye disease. Invest Ophthalmol Vis Sci. 2000;41:961-964.

4. Zhang WS, Prausnitz MR, Edwards A. Model of transient drug diffusion across cornea. J Control Release. 2004;99:241-258.

5. Davis JL, Gilger BC, Robinson MR. Novel approaches to ocular drug delivery. Curr Opin Mol Ther. 2004;6:195-205.

6. Kim JW, Lindsey JD, Wang N, Weinreb RN. Increased human scleral permeability with prostaglandin exposure. Invest Ophthalmol Vis Sci. 2001;42:1514-1521.

7. Ambati J, Canakis CS, Miller JW, et al. Diffusion of high molecular weight compounds through sclera. Invest Ophthalmol Vis Sci. 2000;41:1181-1185.

8. Okabe K, Kimura, Okabe J, et al. Effect of benzalkonium chloride on transscleral drug delivery. Invest Ophthalmol Vis Sci. 2005;46: 703-708.

9. Kim TW, Lindsey JD, Aihara M, Anthony TL, Weinreb RN. Intraocular distribution of 70 -kDa dextran after subconjunctival injection in mice. Invest Ophthalmol Vis Sci. 2002;43:1809-1816.

10. Lindsey JD, Weinreb RN. Identification of the mouse uveoscleral outflow pathway using fluorescent dextran. Invest Ophthalmol Vis Sci. 2002;43:2201-2205.

11. Prausnitz MR, Noonan JS. Permeability of cornea, sclera, and conjunctiva: a literature analysis for drug delivery to the eye. J Pharm Sci. 1998;87:1479-1488.

12. Kim H, Lizak MJ, Tansey G, et al. Study of ocular transport of drugs released from an intravitreal implant using magnetic resonance imaging. Ann Biomed Eng. 2005;33:150-164.

13. Li SK, Jeong EK, Hastings MS. Magnetic resonance imaging study of current and ion delivery into the eye during transscleral and transcorneal iontophoresis. Invest Ophthalmol Vis Sci. 2004;45: $1224-1231$.

14. Huang D, Swanson EA, Lin CP, et al. Optical coherence tomography. Science. 1991;254:1178-1181.

15. Fercher AF, Drexler W, Hitzenberger CK, Lasser T. Optical coherence tomography-principles and applications. Rep Prog Phys. 2003;66:239-303.

16. Podoleanu AG. Optical coherence tomography. Br J Radiol. 2005; 78:976-988.

17. Boppart SA. Optical coherence tomography-principles, applications and advances. Minerva Biotec. 2004;16:211-237.

18. Storen T, Royset A, Svaasand LO, Lindmo T. Measurement of dye diffusion in scattering tissue phantoms using dual-wavelength lowcoherence interferometry. J Biomed Opt. 2006;11:14-17.

19. Ghosn M, Tuchin VV, Larin KV. Depth-resolved monitoring of glucose diffusion in tissues by using optical coherence tomography. Optics Lett. 2006;31:2314-2316.

20. Tuchin VV, Maksimova IL, Zimnyakov DA, Kon IL, Mavlyutov AH, Mishin AA. Light propagation in tissues with controlled optical properties J Biomed Optics. 1997;2:401-417.

21. Esenaliev RO, Larin KV, Larina IV, Motamedi M. Noninvasive monitoring of glucose concentration with optical coherence tomography. Opt Lett. 2001;26:992-994.

22. Larin KV, Eledrisi MS, Motamedi M, Esenaliev RO. Noninvasive blood glucose monitoring with optical coherence tomography-a pilot study in human subjects. Diabetes Care. 2002;25:2263-2267.

23. Larin KV, Motamedi M, Ashitkov TV, Esenaliev RO. Specificity of noninvasive blood glucose sensing using optical coherence tomography technique: a pilot study. Phys Med Biol. 2003;48:13711390.

24. Yeh AT, Hirshburg J. Molecular interactions of exogenous chemical agents with collagen-implications for tissue optical clearing (review). J Biomed Opt. 2006;11:014003.

25. Tuchin VV. Optical Clearing of Tissues and Blood. Bellingham, MA: SPIE Press; 2005. 
26. Tuchin VV. Optical clearing of tissues and blood using the immersion method. J Phys D-Appl Phys. 2005;38:2497-2518.

27. Graaff R, Aarnoudse JG, Zijp JR, et al. Reduced light-scattering properties for mixtures of spherical particles-a simple approximation derived from Mie calculations. Appl Opt. 1992;31:1370-1376.

28. Larin KV, Akkin T, Esenaliev RO, Motamedi M, Milner TE. Phasesensitive optical low-coherence reflectometry for the detection of analyte concentrations. Appl Opt. 2004;43:3408-3414.

29. Kinnunen M, Myllylä R, Jokela T, Vainio S. In vitro studies toward noninvasive glucose monitoring with optical coherence tomography. Appl Opt. 2006; 45:2251-2260.

30. Wang RKK, Xu XQ, Tuchin VV, Elder JB. Concurrent enhancement of imaging depth and contrast for optical coherence tomography by hyperosmotic agents. J Opt Soc Am B-Opt Phys. 2001;18:948953.

31. Tuchin VV, Xu XQ, Wang RK. Dynamic optical coherence tomography in studies of optical clearing, sedimentation, and aggregation of immersed blood. Appl Opt. 2002;41:258-271.

32. Grass GM, Robinson JR. Mechanisms of corneal drug penetration, I: in vivo and in vitro kinetics. J Pharm Sci. 1988;77:3-14.

33. Ke TL, Cagle G, Schlech B, Lorenzetti OJ, Mattern J. Ocular bioavailability of ciprofloxacin in sustained release formulations. $J$ Ocul Pharmacol Ther. 2001;17:555-563.

34. Larin KV, Ghosn M. Influence of experimental conditions on drug diffusion in cornea. Quantum Electronics. 2006;36:1083-1088.

35. Li SK, Molokhia SA, Jeong EK. Assessment of subconjunctival delivery with model ionic permeants and magnetic resonance imaging. Pharm Res. 2004;21:2175-2184.

36. Bashkatov AN, Genina EA, Sinichkin YP, Kochubei VI, Lakodina NA, Tuchin VV. Estimation of the glucosa diffusion coefficient in human eye sclera. Biophysics. 2003;48:292-296.

37. Pawar PK, Majumdar DK. Effect of formulation factors on in vitro permeation of moxifloxacin from aqueous drops through excised goat, sheep, and buffalo corneas. AAPS PharmSciTech. 2006;7: E13.

38. Pfister RR, Burstein N. The effects of ophthalmic drugs, vehicles, and preservatives on corneal epithelium: a scanning electron microscope study. Invest Ophthalmol. 1976;15:246-259.

39. Rohde BH, Chiou GC. Effect of permeation enhancers on betaendorphin systemic uptake after topical application to the eye. Ophthalmic Res. 1991;23:265-271.
40. Vizarova K, Bakos D, Rehakova M, Macho V. Modification of layered atelocollagen by ultraviolet irradiation and chemical crosslinking: structure stability and mechanical properties. Biomaterials. 1994;15:1082-1086.

41. deBoer JF, Milner TE, van Gemert MJC, Nelson JS. Two-dimensional birefringence imaging in biological tissue by polarizationsensitive optical coherence tomography. Opt Lett. 1997;22:934936.

42. Drexler W, Stamper D, Jesser C, et al. Correlation of collagen organization with polarization sensitive imaging of in vitro cartilage: implications for osteoarthritis. J Rheumatol. 2001;28: 1311-1318

43. Jiao SL, Yu WR, Stoica G, Wang LHV. Optical-fiber-based Mueller optical coherence tomography. Opt Lett. 2003;28:1206-1208.

44. Applegate BE, Yang CH, Rollins AM, Izatt JA. Polarization-resolved second-harmonic-generation optical coherence tomography in collagen. Opt Lett. 2004;29:2252-2254.

45. Farrell RA, McCally RL. Corneal transparency. In: Albert DM, Jakobiec FA, eds. Principles and Practice of Ophthalmology. Philadelphia, PA: WB Saunders; 2000.

46. Elliott GF, Goodfellow JM, Woolgar AE. Swelling studies of bovine corneal stroma without bounding membranes. J Physiol. 1980; 298:453-470.

47. Hodson S, Kaila D, Hammond S, Rebello G, al-Omari Y. Transient chloride binding as a contributory factor to corneal stromal swelling in the ox. J Physiol. 1992;450:89-103.

48. Maurice DM. The cornea and the sclera. In: Davson $\mathrm{H}$, ed. The Eye. New York: Academic Press; 1969.

49. Boubriak OA, Urban JP, Akhtar S, Meek KM, Bron AJ. The effect of hydration and matrix composition on solute diffusion in rabbit sclera. Exp Eye Res. 2000;71:503-514.

50. Komai Y, Ushiki T. The three-dimensional organization of collagen fibrils in the human cornea and sclera. Invest Ophthalmol Vis Sci. 1991;32:2244-2258.

51. Jiang J, Geroski DH, Edelhauser HF, Prausnitz MR. Measurement and prediction of lateral diffusion within human sclera. Invest Ophthalmol Vis Sci. 2006;47:3011-3016.

52. Lyubovitsky JG, Spencer JA, Krasieva TB, Andersen B, Tromberg BJ. Imaging corneal pathology in a transgenic mouse model using nonlinear microscopy. J Biomed Opt. 2006;11:14013. 